\title{
Controversias en el manejo clínico de la enfermedad de Whipple
}

\author{
E. OTÓN NIETO, A. CANO RUIZ, M. A. MARTÍN SCAPA, E. NAVAS ELORZA', \\ N. CAMARASA LILLO ${ }^{2}$, A. PALMEIRO URIACH ${ }^{2}$

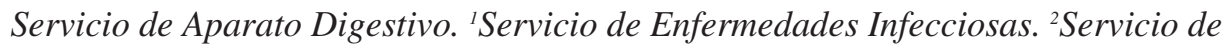 \\ Anatomía Patológica. Hospital Ramón y Cajal. Madrid
}

CONTROVERSIES ABOUT THE CLINICAL MANAGEMENT OF
WHIPPLE'S DISEASE

\begin{abstract}
RESUMEN
Fundamento: Existen muchas controversias relacionadas con el manejo diagnóstico y terapéutico de la enfermedad de Whipple.

Pacientes: Se presentan cuatro casos de enfermedad de Whipple, recogidos del Servicio de Gastroenterología del Hospital Ramón y Cajal de Madrid. La edad media fue 69 años. Los síntomas de presentación fueron en orden de frecuencia artralgias, pérdida de peso, artritis, diarrea, fiebre, dolor epigástrico y síntomas neurológicos. Tres fueron tratados con trimetroprim-sulfametoxazol y uno con cefixima. No hubo recidivas.

Conclusiones: El aspecto endoscópico del duodeno es decisivo para la sospecha de la enfermedad. El tratamiento inicial parenteral de la enfermedad debe realizarse con ceftriaxona e intentar evitar la estreptomicina por su toxicidad, seguido del trimetroprim-sulfametoxazol oral. Como alternativa la cefixima parece eficaz. Los hallazgos típicos de la biopsia pueden persistir meses y años después de finalizado el tratamiento, lo cual resta valor al seguimiento endoscópico de los pacientes tras el tratamiento.
\end{abstract}

PALABRAS CLAVE: Enfermedad de Whipple. Tratamiento. Desnutrición.

\section{ABSTRACT}

Background: There are a lot of controversies related to the diagnostic and therapeutic management of Whipple's disease.

Patients: We present 4 cases of Whipple's disease, collected from the Gastroenterology Service of the Hospital Ramon y Cajal, from Madrid.

Results: The average age was 69. The first symptoms were in order of frequency, arthralgias, loss of weight, arthritis, diarrhoea, fever, epigastric pain and neurologic symptoms. Three were treated with trimethoprimsulphamethoxazol and one with cefixime. There were no recurrences.

Conclusions: The endoscopic appearance of the duodenum is fundamental for the suspicion of the disease. The initial parenteral treatment may consist of ceftriaxone and try to avoid using streptomycine because of its toxicity, followed by oral trimethoprim-sulphamethoxazol. As an alternative to the latter, cefixime is usually effective. The typical findings of the duodenum biopsy can persist several months or years after finishing the treatment, decreasing the value of endoscopic pursuit after treatment.

KEY WORDS: Whipple's disease.Treatment. Malnutrition.

E. Otón Nieto, A. Cano Ruiz, M. A. Martín Scapa, E. Navas Elorza, N. Camarasa Lillo, A. Palmeiro Uriach. Controversias en el manejo clínico de la enfermedad de Whipple. An Med Interna (Madrid) 2004; 21: 488-490.

\section{INTRODUCCIÓN}

La enfermedad de Whipple es una rara enfermedad multisistémica, de etiología infecciosa que con frecuencia comienza con manifestaciones clínicas digestivas y se acompaña habitualmente de un abanico de síntomas diversos que puede mimetizar muchas otras entidades clínicas, planteándose un amplio diagnóstico diferencial. El conocimiento del agente infeccioso implicado, Tropheryma whipplei, ha supuesto una revolución terapéutica, convirtiéndose en una entidad relativamente fácil de tratar, cuando antaño era mortal en casi todos los casos. El objetivo de este trabajo es la descripción de la casuística del Servicio de Gastroenterología del Hospital Ramón y Cajal de Madrid entre los años 1992 y 2003, con especial énfasis en las características clínicas más peculiares.

\section{CASOS APORTADOS}

Caso 1: varón de 74 años con historia de pérdida de peso de $10 \mathrm{~kg}$ en los últimos 10 meses así como artralgias de hombros y rodillas. Había presentado brotes de diarrea episódica sin productos patológicos con astenia, anorexia y febrícula. A la exploración se advirtió una hiperpigmentación de la piel y desnutrición importante. En la analítica básica destacaba una anemia microcítica, eosinofilia en sangre, veloci-

Trabajo aceptado: 15 de abril de 2004 
dad de sedimentación globular (VSG) 57 seg, albúmina 2,46 g/dl, colesterol 82mg/dl, sideremia $12 \mathrm{mcg} / \mathrm{dl}$, ferritina $12 \mathrm{ng} / \mathrm{ml}$, Van de Kamer 9,2 g de grasa/día. La tomografía axial computerizada (TAC) mostró gran cantidad de adenopatías retroperitoneales y mesentéricas de tamaño significativo. En la endoscopia oral se observó una imagen compatible con gastritis crónica y un punteado blanquecino en segunda porción duodenal. La biopsia duodenal demostró macrófagos positivos al ácido peryódico de Schiff (PAS) y tinción de Ziehl negativa, compatible con la enfermedad de Whipple (Fig. 1). El paciente se empezó a tratar con penicilina y estreptomicina 15 días y luego trimetroprim-sulfametoxazol (TM-SF) pero presentó una reacción urticarial, por la cual hubo que suspender este último, pasando a doxiciclina. Tuvo mejoría clínica y aumentó de peso, pero al persistir los macrófagos PAS positivos en la biopsia, se repiten hasta dos ciclos más de 15 días de penicilina y estreptomicina. En la evolución tuvo un cuadro de inestabilidad de la marcha y sensación de mareo secundarios a toxicidad laberíntica por estreptomicina, que inicialmente planteó diagnóstico diferencial con afectación neurológica de la enfermedad. Se retiró entonces la doxiciclina, por asociarse a mayor número de recidivas, y se trató con cefixima. El paciente cumplió dos años de tratamiento antibiótico estando asintomático en la actualidad.

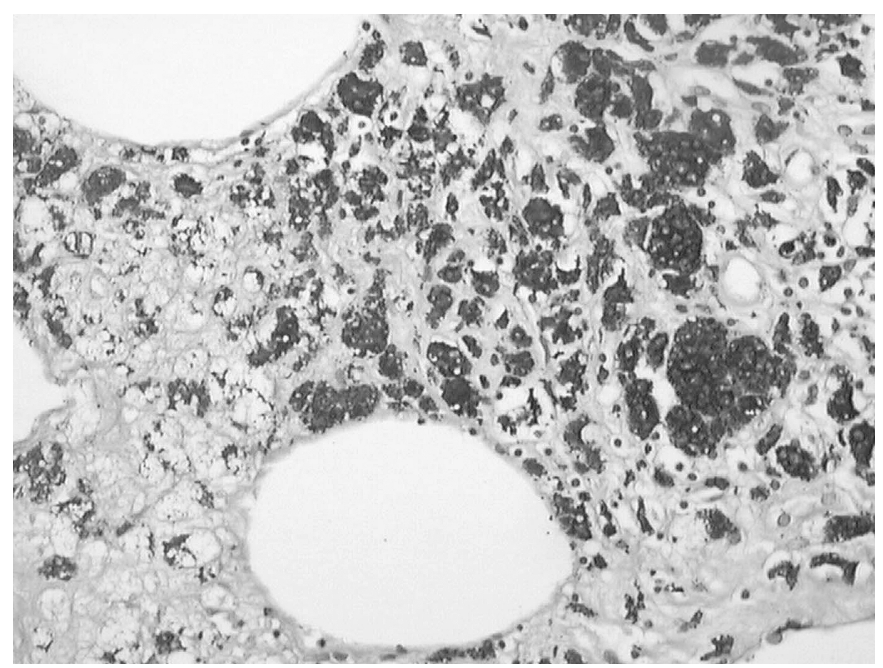

Fig. 1. Imagen de microscopio óptico de biopsia duodenal con macrófagos con tinción PAS positiva ocupando la lámina propia intestinal. Quistes de grasa extravasada (aumentos originales $x$ 400). Cedida por el Servicio de Anatomía Patológica del Hospital Ramón y Cajal de Madrid.

Caso 2: varón de 54 años con historia desde hacía dos años de poliartritis simétrica seronegativa con brotes de afectación en hombros, codos, carpos, rodillas, tobillos, y metacarpo-falángicas. Fue tratado con dosis variables de corticoides, antiinflamatorios no esteroideos (AINE) y metotrexate, en el servicio de Reumatología. El paciente ingresa por presentar epigastralgia intensa, fiebre alta de hasta $39^{\circ}$, pérdida de peso de $6 \mathrm{~kg}$ y malestar general en los últimos dos meses. En la exploración física presentaba febrícula de $37,6^{\circ}$, adenopatías cervicales rodaderas no dolorosas y hemiabdomen superior doloroso a la palpación. En la analítica tenía leucocitosis de $17,3 \times 10^{3} / \mathrm{mcl}$, y proteínas totales de 5,4 g/dl con Albúmina de 2,66 g/dl. La grasa en heces era de $3,8 \mathrm{~g} / 24 \mathrm{~h}$. En la TAC se observaban numerosas adenopatías algunas mayores de $2 \mathrm{~cm}$. La biopsia de un ganglio mesentérico por laparoscopia mostró macrófagos PAS positivos. Se realizó endoscopia oral con biopsia duodenal que confirmó el diagnóstico. Se trató inicialmente con ceftriaxona y estreptomicina durante 15 días y posteriormente con TMSF. A los 6 meses de tratamiento, había presentado franca mejoría del cuadro, persistiendo artralgias sin artritis. Continúa bajo tratamiento en la actualidad.
Caso 3: mujer de 75 años que acudió por presentar desde hacía un año deposiciones líquidas sin productos patológicos con pérdida de 13 $\mathrm{kg}$ de peso, astenia y artralgias con artritis de muñeca derecha y rodillas. Estaba diagnosticada de artropatía por pirofosfato y artrosis. Presentaba además cuadros de mareo inespecífico e inestabilidad de la marcha. En la exploración física tenía malnutrición importante, edemas de miembros inferiores, limitación a la mirada superior, discreta asimetría facial y marcha inestable con flexión ventral del tronco. En la analítica tenía anemia microcítica y una albúmina de $3,0 \mathrm{~g} / \mathrm{dl}$ sin otras alteraciones. Grasa en heces de 11,2 g/día. El tránsito gastrointestinal mostraba dilatación de asas de intestino delgado con imagen sugerente de malabsorción. En la endoscopia oral se objetivaron unas placas amarillentas en segunda porción duodenal que en la biopsia fueron compatibles con enfermedad de Whipple. Fue tratada con penicilina G y estreptomicina dos semanas y después con TM-SF y al año de tratamiento la paciente había ganado $8 \mathrm{~kg}$, había desaparecido la diarrea y había mejorado de forma importante la inestabilidad. En la biopsia duodenal tras el tratamiento las zonas PAS positivas eran ya amorfas y al microscopio electrónico se veían membranas degeneradas sin bacilos viables (Fig. 2). Se suspendió el tratamiento.

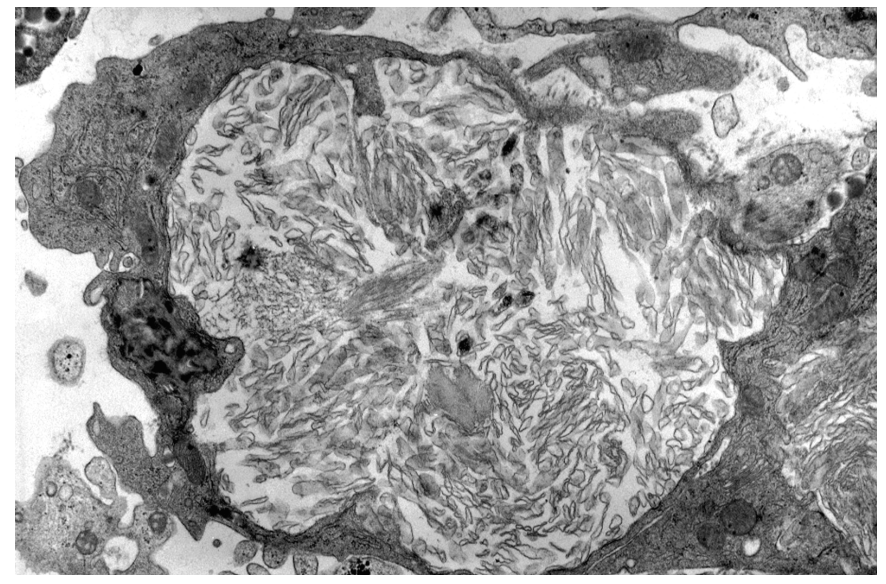

Fig. 2. Imagen de microscopio electrónico de biopsia duodenal de una enfermedad de Whipple con respuesta completa a tratamiento. Restos de membranas bacterianas degeneradas. Cedida por el Servicio de Anatomía Patológica del Hospital Ramón y Cajal.

Caso 4: mujer de 73 años con pérdida de peso de $10 \mathrm{~kg}$, astenia y anorexia, así como diarrea acuosa, de 4 meses de duración. Desde hace 15 días presentaba además dolor epigástrico y artralgias en muñecas, hombros y tobillos. En la exploración tenía hiperpigmentación, datos de desnutrición y signos de artritis en muñeca izquierda. En la analítica presentaba anemia microcítica y niveles bajos de albúmina. El tránsito gastrointestinal mostraba asas intestinales dilatadas y floculación del contraste. No se objetivaron adenopatías en la TAC. En la endoscopia oral se objetivaron formaciones blanquecinas múltiples y difusas compatibles con linfangiectasias. La anatomía patológica mostró la clásica lipodistrofia intestinal con macrófagos PAS positivos y formas bacilares características en el microscopio electrónico. Se le trató con TM-SF durante un año, pero al persistir el material PAS positivo en la biopsia, se continúo el tratamiento hasta completar dos años. La paciente recuperó el peso perdido y desapareció la sintomatología pero la biopsia no se normalizó hasta dos años después de finalizado el tratamiento.

\section{DISCUSIÓN}

Epidemiológicamente se ha descrito en las series más antiguas un claro predominio masculino en la enfermedad de 
Whipple, llegando a hablar de incidencia 8 veces mayor en varones. Sin embargo desde 1991 asistimos a un aumento en el porcentaje de mujeres afectas de hasta un $20 \%$ (1) o incluso porcentajes mayores (2). De nuestros casos, la mitad correspondía a mujeres, y en general parece haber en la literatura un ascenso de la incidencia en el sexo femenino que no tiene una explicación clara. Asimismo en la literatura encontramos una media de edad al diagnóstico de alrededor de 50 años $(1,3)$, que es claramente mayor que en décadas previas como demuestra von Herbay y cols. (2). De nuestros pacientes, 3 presentaban más de 70 años, lo cual va a favor de este incremento en la edad del debut, que en ocasiones se ha vinculado al uso masivo de antibióticos en la sociedad actual (1).

La enfermedad de Whipple se caracteriza por el pleomorfismo de sus manifestaciones clínicas, siendo las artralgias la forma de presentación más frecuente $(67-75 \%$ de los casos) $(4,5)$. No es raro que el diagnóstico se demore varios meses o incluso años (6) en las formas de presentación más atípica. Llama la atención el caso 2 en el cual el paciente presentó solamente clínica articular durante dos años y fue politratado con corticoides, antiinflamatorios y metotrexate con mejoría parcial, hasta que la aparición de nuevos síntomas orientó el diagnóstico. Sin embargo la afectación neurológica previa al diagnóstico es rara y no supera el $4 \%(4,7)$. El caso 3 ya presentaba síntomas de inestabilidad en la marcha y oftalmoplejia en el momento del diagnóstico de la enfermedad. En estos casos, probablemente la utilización del TM-SF y en un caso de la cefixima, permitieron la curación completa sin recidiva neurológica, más frecuente con el uso de doxiciclina (3).

Un dato diagnóstico importante de los casos presentados y al cual no siempre se hace mención, es el aspecto endoscópico típico del duodeno con un punteado blanquecino difuso compatible con linfangiectasias. Esto debe hacer al endoscopista sospechar la enfermedad y realizar biopsias múltiples.

El tratamiento de elección en la actualidad es el TM-SF que se debe administrar junto con ácido fólico (8). No son raras las alergias a este antibiótico (caso 1) y hay varias alternativas como la penicilina oral, cefixima,, fluorquinolonas, etc. (3). Las tetraciclinas están cayendo en desuso por las tasas de recidiva claramente mayores debido a que no traspasan bien la barrera hematoencefálica, no debiendo utilizarse en primera intención $(9,10)$. Las pautas parenterales iniciales de tratamiento se reservan para los casos más severos, e incluso en estos casos se debe intentar evitar la estreptomicina que es ototóxica (caso 1) hasta en un 5\% de casos y nefrotóxica en 5$20 \%$ de casos(11). En el caso 1, además de producir toxicidad vestibular, hizo pensar primeramente en una recidiva neurológica de la enfermedad, por la clínica de inestabilidad en la marcha. En su lugar podría recomendarse la ceftriaxona como pauta parenteral inicial (3).

Un aspecto controvertido de esta enfermedad es el seguimiento endoscópico de los pacientes tratados. Según algunos autores $(3,12)$, se debe realizar endoscopia oral cada 6 meses para observar la desaparición del material PAS positivo y considerar así al paciente curado. Sin embargo, tres de nuestros casos tienen ya un seguimiento de varios años tras el tratamiento y en los tres, persistió el material PAS positivo, cuando el paciente ya había cumplido al menos 1 año o más de tratamiento y se encontraba totalmente asintomático. Dicho material podría corresponder sólo a bacilos muertos que no serían indicativos de posibles recidivas siendo entonces la visión anatomopatológica insuficiente para este propósito. Asimismo también otros autores (13-16) apoyan esta observación y recomiendan realizar la técnica PCR sobre las biopsias duodenales aumentando el valor predictivo de recidiva. Probablemente si no se dispone de esta técnica, la realización de endoscopia oral cada 6 meses no tendría lugar (17), puesto que puede dar lugar a prolongaciones injustificadas de tratamiento antibiótico, en pacientes que por lo demás se encuentran ya asintomáticos. Existen casos en los que la imagen del microscopio electrónico puede ser diagnóstica de curación y es así cuando sólo se observan membranas bacterianas desestructuradas sin estructuras bacilares delimitadas (Fig. 2). Sin embargo en muchas ocasiones se observan imágenes intermedias de bacilos de dudosa viabilidad que hacen difícil dictaminar la curación completa.

\section{Bibliografía}

1. Dutly F, Altwegg M. Whipple's disease and Tropheryma whippelii. Clin Microbiol Rev 2001; 14: 561-583.

2. Von Herbay A, Otto HF, Stolte M, Borchard F, Kirchner T, Ditton HJ, et al. Epidemiology of Whipple's disease in Germany. Analysis of 110 patients diagnosed in 1965-95. Scand J Gastroenterol 1997; 32: 52-57.

3. Marth T, Raoult D. Whipple's disease. Lancet 2003; 361: 239-246.

4. Durand DV, Lecomte C, Cathebras P, Rousset H, Godeau P. Whipple Disease. Clinical Review of 52 cases. Medicine 1997; 76: 170-184.

5. Puechal X. Whipple's disease. Joint Bone Spine 2002; 69: 133-140.

6. Caples SM, Petrovic LM, Ryu JH. Mayo Clin Proc 2001; 76 (10): 1063-1066.

7. Peters G, du Plessis DG, Humphrey PR. Cerebral Whipple's disease with stroke-like presentation and cerebrovascular pathology. J Neurol Neurosurg Psychiatry 2002 Sep; 73 (3): 336-339.

8. Ratnaike RN. Whipple's disease. Postgrad Med J 2000; 76: 760-766.

9. Singer R. Diagnosis and Treatment of Whipple's Disease. Drugs 1998; 55: 699-704.

10. Keinath RD, Merrell DE, Lietstra RV, Dobbins WO. Antibiotic treatment and relapse in Whipple's disease. Gastroenterology 1985; 88: 1967-1973.
11 Flórez J, Armijo JA, Mediavilla A, editors. Farmacología Humana. Segunda edición. Barcelona, 1992.

12. Müller N, Schneider T, Zeitz M, Marth T. Whipple's disease: new aspects in pathogenesis and diagnosis. Acta Endoscopica 2001, 31: 243-253.

13. Ramzan NN, Loftus E, Burgart LJ, Rooney M, Batts KP, Wiesner RH, et al. Diagnosis and Monitoring of Whipple Disease by Polymerase Chain Reaction. Ann Intern Med 1997; 126: 520-527.

14. Maiwald M, Relman DA. Whipple's disease and Tropheryma whippelii: Secrets Slowly Revealed. CID 2001; 32: 457-463.

15. Jirout Casillas F, Ballina García FJ, Fernández Sánchez JA, Queiro Silva R, Ordás Calvo C, Rodríguez Pérez A. Manifestaciones atípicas de la enfermedad de Whipple. An Med Interna (Madrid) 2001; 18: 24-26.

16. Wallace MB, Apstein MD. Whipple's disease. Uptodate [revista electrónica] 2003; 11 (2) [consultado 01-08-2003]: Disponible en http://www.uptodate.com.

17. Gómez de la Torre IJ, Claros González C, López Muñiz C, Velasco Alvarez A. Enfermedad de Whipple: diagnóstico temprano a través de patología articular e hiperpigmentación. An Med Interna (Madrid) 1998; 15: 53-55. 